\title{
Politiques de non-intégration dans les monarchies du Golfe
}

Discuter les raisons de leur pérennité

Non-integration policies in the Gulf monarchies. The reasons behind their longevity

\section{Claire Beaugrand}

\section{OpenEdition}

\section{Journals}

Édition électronique

URL : http://journals.openedition.org/transcontinentales/793

DOI : 10.4000/transcontinentales.793

ISBN : 978-2-7351-1557-0

ISSN : 1775-397X

Éditeur

Editions de la maison des sciences de l'homme

Édition imprimée

Date de publication : 31 décembre 2010

ISSN : 1950-1684

\section{Référence électronique}

Claire Beaugrand, «Politiques de non-intégration dans les monarchies du Golfe », Transcontinentales [En ligne], 8/9 | 2010, document 8, mis en ligne le 31 décembre 2010, consulté le 08 septembre 2020 URL : http://journals.openedition.org/transcontinentales/793 ; DOI : https://doi.org/10.4000/ transcontinentales.793

Ce document a été généré automatiquement le 8 septembre 2020

Tous droits réservés 


\title{
Politiques de non-intégration dans les monarchies du Golfe
}

\author{
Discuter les raisons de leur pérennité \\ Non-integration policies in the Gulf monarchies. The reasons behind their \\ longevity
}

Claire Beaugrand

1 En avril 2007, dans un article publié par le quotidien anglophone Arab Times, le ministre koweïtien du Travail et des Affaires sociales indiquait que le terme « d'immigrant » ne saurait être employé dans la législation émanant de son ministère. Il lui préférait celui plus approprié, « d'expatrié », niant ainsi la réalité et la possibilité d'une implantation à long terme des travailleurs étrangers résidant dans l'Émirat ${ }^{1}$.

2 L'absence d'intégration des travailleurs migrants est, à l'heure actuelle, une des caractéristiques les plus marquantes des monarchies du Conseil de coopération du Golfe (ccG : Arabie Saoudite, Bahreïn, Émirats arabes unis, Koweït, Qatar et Oman). Pays très peu peuplés lorsqu'ils commencent leur décollage économique dans les années 1950-1960, les pays du CCG ont fait massivement appel aux travailleurs immigrés pour répondre à leurs besoins de main-d'œuvre. Pour les frères arabes qui se sont installés les premiers aux beaux jours du nationalisme arabe, la question de l'intégration des migrants a pu se poser. Cependant, les tendances antimonarchiques de l'idéologie nationaliste arabe orientèrent bientôt les dirigeants vers des politiques migratoires visant, d'une part, à garder le nombre de citoyens relativement limité et, d'autre part, à isoler les nationaux des influences des travailleurs étrangers. L'établissement de hiérarchies professionnelles sur la base de la nationalité, avec les nationaux aux postes de direction, puis le recours aux contrats à durée limitée et à la rotation de main-d'œuvre, le plus souvent asiatique, servirent ce double objectif.

3 Le résultat de ces politiques de non-intégration des populations immigrées est bien connu. Il se manifeste par le fait que, dans certains pays du $\mathrm{CCG}$, la présence étrangère l'emporte en nombre sur la population nationale. En 2005, d'après le Programme des Nations unies pour le développement (PNUD) ${ }^{2}$, les pourcentages d'étrangers au sein de la 
population des pays du CCG étaient les suivants : 80,5\% au Qatar, $70 \%$ aux Émirats arabes unis (EAU), $69,2 \%$ au Koweït, et $25,5 \%$ en Oman, $26,8 \%$ en Arabie Saoudite et $38,2 \%$ au Bahreïn. Ces pourcentages sont encore plus déséquilibrés si l'on regarde la proportion d'étrangers au sein de la population active, et atteignent des ratios de neuf étrangers pour un national si l'on se concentre sur la composition des employés du secteur privé.

4 En outre, les pays du CCG comptent aujourd'hui avec Singapour et Hong Kong parmi les pays où les perspectives d'intégration et d'implantation permanente des travailleurs étrangers sont les plus faibles (Ruhs \& Martin 2008 : 257).

Depuis la seconde guerre du Golfe, en 1991, en dépit des pressions des ONG occidentales prônant l'octroi de plus de droits et de libertés aux nationaux et, de plus en plus, aux migrants, le modèle migratoire golfien a perduré. Il a également résisté aux pressions intérieures liées à la détérioration du marché national de l'emploi et à l'accroissement du taux de chômage parmi les nouvelles générations de citoyens. À l'heure actuelle, il se caractérise toujours par un système de recrutement massif sur une base temporaire, avec des permis de résidence attachés à un seul employeur ${ }^{3}$ et des droits économiques et sociaux limités pour les migrants ${ }^{4}$.

6 Analyser la pérennité et évaluer la durabilité du système golfien de migration en le replaçant dans son contexte international, tels sont les buts de cet article à travers principalement mais non exclusivement le cas du Koweït ${ }^{5}$.

7 Dans un premier temps, nous étudierons la mise en place du système de nonintégration. Nous identifierons les deux raisons principales qui ont conduit à éliminer l'option de l'intégration des migrants arabes et à adopter un modèle de non-intégration fondé sur des migrations temporaires. La première tient au fait que, depuis leur indépendance, les pays du Golfe, en particulier les cités-États, ne peuvent assurer leur sécurité et garantir leur souveraineté par eux-mêmes. Cela les rend particulièrement vulnérables, y compris dans le domaine crucial des migrations, aux influences extérieures, et explique l'impact qu'a eu le nationalisme arabe sur leurs politiques migratoires.

8 La deuxième raison tient aux impératifs de survie du régime monarchique lui-même. En effet, les familles royales golfiennes tirent leur légitimité de l'octroi de privilèges à leurs populations nationales et, dans le domaine particulier qui nous intéresse, des bénéfices que ces dernières peuvent retirer de la présence d'étrangers dans le pays.

En conservant ces deux paramètres en tête, nous évaluerons, dans un deuxième temps, la durabilité des politiques de non-intégration. Pour cela, nous prendrons en compte, d'une part, l'impact de l'environnement international marqué depuis vingt ans par le libéralisme économique et, d'autre part, l'évolution des économies du ccG.

\section{Mise en place du système de non-intégration}

10 L'histoire des migrations vers le Golfe est relativement bien documentée grâce aux travaux de trois économistes (Seccombe 1985). En 1977-1978, Birks et Sinclair ont mené une vaste enquête sur les migrations économiques dans le cadre d'un programme de la Banque mondiale (Birks, Seccombe et Sinclair 1988). Ian Seccombe, quant à lui, a largement contribué aux études sur les migrations golfiennes en les réinscrivant dans la perspective historique (Seccombe 1983 ; Seccombe et Lawless 1986). Il s'agit moins ici 
de retracer l'histoire de ces migrations que de mettre au jour les raisons qui ont conduit à l'adoption d'un système de recrutement temporaire strict d'une maind'œuvre majoritairement asiatique pour remplacer les migrants arabes de plus long terme.

11 Tous les analystes de la région ont noté, chiffres à l'appui, la rupture qui s'est opérée dans la composition par origine du recrutement des travailleurs immigrés au tournant des années 1980 (Abella 1995 ; Kapiszewski 2006 ; Russell 1990). Alors que, jusque-là, la majorité de la population étrangère provenait des pays arabes, Égypte, Yémen et Palestine/Jordanie en tête, ce sont désormais les ressortissants du subcontinent indien et de l'Asie du Sud-Est (Corée du Sud, Thaïlande, Philippines, Indonésie) qui constituent le groupe d'expatriés le plus nombreux.

12 Ces migrants, d'origine lointaine et qui ne partagent ni la langue ni la culture arabes, sont venus avec un très faible désir, et aussi peu de chances, de s'installer durablement. Jusque-là, la ségrégation entre nationaux et étrangers avait principalement reposé sur la différence de droits socioéconomiques entre les deux catégories, sur une hiérarchie sociale et une organisation urbaine fondées sur la base de la nationalité et scellées par le blocage, de facto,des voies de naturalisation en dépit des lois les prévoyant.

13 Soucieux de préserver le petit nombre et la loyauté de leurs ressortissants, les régimes du Golfe avaient dans les années 1960-1970 remporté une victoire sur les nationalistes arabes en éloignant toute possibilité de naturalisation de droit pour les migrants arabes. À Koweït, par exemple, cela s'était traduit par des exigences toujours renouvelées en termes de nombre d'années de résidence dans le pays (de huit à quinze ans pour les ressortissants de pays arabes) et par la définition d'un chiffre annuel maximal de naturalisations ( 50 par an), elle-même limitée par le caractère discrétionnaire en dernier ressort de la procédure d'octroi de la nationalité - cette dernière caractéristique valant pour tous les pays du Golfe.

Le traitement différencié n'avait pourtant pas empêché que l'intégration des communautés arabes se produise assez naturellement dans de nombreux domaines (Russell 1990). La formule qui consistait à importer du travail tout en évitant la formation d'une classe laborieuse revendicative commençait à montrer ses limites (Beaugé et Roussillon 1988 ; Fargues 2000 ; Longuenesse, Beaugé et Nancy 1986).

15 La raison la plus communément avancée pour expliquer le changement de politique migratoire et le choix d'un recrutement temporaire de main-d'œuvre asiatique est la menace que les ressortissants arabes plus politisés que leurs frères du Golfe faisaient peser sur les régimes monarchiques.

16 Les troubles liés à la promotion d'un islam politique radical chiite ont clairement montré l'influence déstabilisatrice que les étrangers pouvaient avoir sur le pays et sur les nationaux, et ce, en dépit de leur ségrégation et de leur absence d'intégration politique. Si les auteurs de la vague d'explosions qui toucha le Koweït, dans les années 1980 sont d'abord des chiites étrangers originaires d'Irak et du Liban, les attentats de la fin de la décennie furent revendiqués par des chiites koweïtiens, issus, pour certains, des grandes familles marchandes de l'émirat. 


\section{Débat initial : réduction, intégration ou rotation des étrangers ?}

17 Les débats publics autour de l'immigration qui agitent le Koweït des années 1980 sont particulièrement éclairants pour rendre compte du tournant pris par les politiques migratoires dans les pays du Golfe. Ils montrent, d'une part, la préoccupation réelle des autorités koweïtiennes pour le déséquilibre démographique entre nationaux et étrangers. Ils révèlent aussi l'impossibilité, pour ces mêmes autorités koweïtiennes, de neutraliser la menace politique représentée par les migrants en réduisant leur nombre. Car, la présence massive d'étrangers profite aux citoyens koweïtiens.

Le choix d'une politique de régulation plus rigide des permis de travail, délivrés pour des durées plus courtes, a ainsi représenté un compromis entre ces deux contraintes. L'adoption du système de rotation des migrations a permis de conserver le nombre élevé des travailleurs étrangers tout en minimisant le risque de revendications et d'activisme.

Boghardt $(2006: 68,87,112)$, qui étudie la politique de sécurité intérieure du Koweït dans ces années de tourmente, relève une contradiction d'importance en ce qui concerne le traitement des travailleurs étrangers durant cette période. Elle note que, en réponse aux premiers attentats terroristes, le gouvernement koweïtien s'est lancé dans des campagnes publiques d'expulsion des résidents illégaux. Pourtant, souligne-telle, ces campagnes n'ont qu'un temps car elles sont largement ostentatoires. Si elles visent avant tout à répondre aux critiques émises contre l'incapacité de la famille royale à faire face aux menaces émanant des réseaux activistes chiites, elles heurtent également des intérêts économiques puissants au sein de la population nationale.

De fait, en plus des profits qu'ils apportent à leurs employeurs et des bénéfices qu'ils partagent avec leurs représentants locaux ${ }^{6}$, les étrangers constituent, par leur présence même, une source de revenus pour les Koweïtiens (Asmar [1990] 1995). Seuls à jouir du droit de propriété immobilière, ces derniers collectent les loyers ; seuls à bénéficier de monopoles d'importations et à gérer les réseaux de distribution, ils profitent de marges sur la consommation de biens et services par tous les résidents. Seuls à être actionnaires, de droit, des coopératives de distribution ${ }^{7}$, ils bénéficient aussi des dividendes.

21 Le débat qui a porté sur une possible réduction structurelle de la présence étrangère, au début des années 1980, a montré l'ampleur du conflit d'intérêts à l'époque. Face aux inquiétudes de la famille royale, certains parlementaires prônaient une politique d'intégration car, pensaient-ils, la naturalisation des résidents arabes de long terme permettrait de garantir leur loyauté (Boghardt 2006 : 83). Clairement cette option leur semblait préférable à la réduction du nombre d'étrangers. Ces hésitations entre politique d'intégration et de non-intégration scindèrent aussi le gouvernement. En 1984, le ministère de l'Intérieur proposait de relever le seuil de revenu au-dessus duquel le regroupement familial était autorisé, afin de réduire, pour des raisons sécuritaires, le nombre d'immigrants arabes sur le territoire koweïtien. Il se heurta à une forte opposition du ministère du Travail et des Affaires sociales, qui entendait, quant à lui, favoriser l'intégration et, surtout, la consommation des migrants déjà établis au Koweït, dans le contexte de récession économique marquant la décennie 1980. 


\section{Migrations temporaires asiatiques et déclin du nationalisme arabe} recrutement de main-d'œuvre asiatique s'est tassé (Birks, Seccombe et Sinclair 1986 Feiler 1991), ce dernier n'a cessé d'augmenter depuis la seconde moitié des années 1980, pour n'être interrompu que par la parenthèse de la seconde guerre du Golfe. L'invasion du Koweït par Saddam Hussein qui se targuait d'une politique de porte ouverte aux immigrants arabes (Roussillon 1985) et l'expulsion du ccG des communautés palestiniennes et yéménites, dont les dirigeants avaient eu foi dans la rhétorique panarabiste du président irakien, enterreront définitivement les possibilités d'intégration des migrants. Privés du support idéologique que constituait le nationalisme arabe, les migrants arabes n'ont depuis lors aucune revendication d'intégration politique ou économique. Ils cultivent, pour la plupart, leurs liens transnationaux ${ }^{8}$, comme une forme de sécurité. La main-d'œuvre asiatique a, quant à elle, trouvé à s'employer dans des secteurs toujours plus diversifiés, de la construction aux services. Elle provient, enfin, de pays où le travail est toujours meilleur marché, comme le Bangladesh ou le Sri Lanka (Abella 1995 : 422).

\section{Durabilité du système de non-intégration des migrants}

La façon dont furent mises en place les politiques de non-intégration a permis d'identifier les contraintes intérieures et extérieures pesant sur l'élaboration et l'application des politiques migratoires golfiennes. Dans cette seconde partie, nous nous attachons à mettre au jour le rôle joué par ces mêmes facteurs explicatifs, mais tels qu'ils se présentent à l'heure actuelle.

Notons, tout d'abord que, si le nationalisme arabe a disparu en tant que force politique prônant un traitement préférentiel pour les migrants arabes, il a laissé un héritage de long terme en ce qui concerne la légitimation des politiques de non-intégration. Le discours de justification des politiques migratoires masque parfois leur réalité, car il s'adresse aussi aux puissances extérieures qui garantissent la sécurité de la région et tâche de se conformer aux normes prévalant chez ces " protecteurs ".

27 Ainsi, alors que le nationalisme anti-impérialiste et le nassérisme battaient leur plein, les dirigeants golfiens légitimèrent l'importation de main-d'œuvre et, surtout, sa nonintégration en la présentant comme une phase transitoire de leurs développements nationaux. Elle permettait de poursuivre une croissance soutenue le temps que la population nationale en plein boom démographique se dote des qualifications 
nécessaires pour en prendre le relais. Cette version officielle permettait aussi de justifier le déséquilibre démographique entre nationaux et immigrés qui allait à l'encontre des normes du nationalisme ethnique dominant dans la région - et $\mathrm{du}$ système international d'États-nations.

\section{D'une justification à l'autre}

28 Cette version officielle n'a pas changé. Dans cette même logique, s'inscrivent les politiques de nationalisation de la main-d'œuvre - saoudisation, omanisation, bahreïnisation, etc. - mises en place depuis plus de vingt ans aujourd'hui, pour tâcher de remédier à la montée du chômage chez les nationaux. Ces politiques ont tenté de remplacer les travailleurs étrangers par des travailleurs nationaux, en encourageant le secteur privé à employer ces derniers suivant un certain quota.

Devenues un leitmotiv de la politique économique, de ses plans de développement, et de la presse nationale ${ }^{9}$, elles ont entretenu l'illusion du caractère temporaire de la présence des travailleurs étrangers. Mais, leurs résultats ont été plus que décevants. Elles se sont heurtées à la résistance des entreprises locales et étrangères, dont les dirigeants confessaient même devoir payer des nationaux à rester chez eux et continuer à employer des étrangers, considérés comme plus fiables.

De fait, ces politiques toujours reconduites en dépit de leurs résultats ont servi autant leurs objectifs proclamés qu'elles ont justifié les politiques discriminatoires de nonintégration dans un contexte normatif international nouveau : car, si les immigrés ont vocation à être, tôt ou tard, remplacés par des nationaux, nul besoin n'est de changer drastiquement les conditions de leur accueil ni leurs conditions de travail.

$31 \mathrm{Du}$ nationalisme arabe au libéralisme et à la mondialisation, l'évolution de l'environnement international n'a pas fait cesser le besoin de justifier les politiques discriminatoires. Elle a seulement modifié la manière dont les États légitiment leurs politiques de non-intégration. L'alliance entre les Etats-Unis et les pays du CCG après la libération du Koweït en 1991 s'est accompagnée de pressions du gouvernement américain pour libéraliser les pays du Golfe dans le domaine économique, mais aussi d'actions dans le domaine des droits et des libertés individuels des organisations de défense des droits de l'homme, arrivées dans la région à la faveur de la seconde guerre du Golfe. La position de Castles (2006: 746), par exemple, offre une interprétation normative fortement ancrée dans le contexte de promotion démocratique qui a marqué les politiques étrangères occidentales des années 2000. Selon lui, le modèle golfien est, tout autant que les programmes de Gastarbeiter des pays occidentaux, voué à l'échec. Il disparaîtra dès que le processus de démocratisation et de libéralisation politique aura fait son œuvre dans la région, car l'octroi progressif de droits aux migrants particulièrement les moins qualifiés qui représentent la majorité des migrants vers le Golfe - viendra relativiser la compétitivité de leurs salaires bas (Castles 1986) ${ }^{10}$. Le précédent créé par le mouvement de grève des travailleurs du bâtiment à Doubaï, en 2006, fournirait, selon cette logique, la preuve de la quasi-impossibilité de limiter indéfiniment les droits des travailleurs étrangers.

C'est plutôt l'inverse qui se produit. Depuis une vingtaine d'années désormais, la tendance ne consiste pas tant à revenir sur des politiques discriminatoires qu'à favoriser et à s'adapter à l'accélération de la mobilité du travail, en en redressant les excès, conformément au régime international des droits de l'homme. 


\section{Évolution libérale de l'organisation mondiale du travail immigré} temporaire qui font l'objet des grèves et des mouvements de protestation des travailleurs étrangers dans le Golfe. Que ce soient les employés de l'entreprise de nettoyage koweïtienne Tanzifco en 2005, ou ceux de l'entreprise du BTP à Dubaï un an plus tard, les migrants protestent contre l'arriéré de paiement de leurs salaires. Leurs mouvements de contestation constituent des actions de dernier recours contre la violation des clauses de leurs contrats et non contre les principes de ségrégation ou d'inégalité, justifiés par le principe de souveraineté territoriale et exacerbés par le fonctionnement sur la base d'une population citoyenne réduite des régimes golfiens. Il en va de même des condamnations annuelles des États du Golfe par le secrétariat d'État américain dans ses «rapports par pays $»^{11}$. S'accommodant des pratiques discriminatoires et des conditions de travail, ces rapports visent avant tout les dérives les plus grossières des politiques de recrutement massif de main-d'œuvre étrangère (peu qualifiée) lorsqu'ils critiquent l'incapacité ou le manque de volonté des pays du CCG à éradiquer les réseaux de trafics de personnes.

\section{Différentes structures économiques, différentes perspectives}

Sur le plan intérieur, les politiques de non-intégration ont en général permis aux régimes de conserver leur support auprès des citoyens qui profitent économiquement de cette présence étrangère, ce qui n'est plus toujours aussi directement le cas. Le système fonctionne sans faille dans les pays où la rente est la plus abondante (Qatar, Koweït) et dans ceux où la bourgeoisie marchande a pu s'imposer dans les affaires aux côtés de la famille royale, comme au Koweït. Dans les émirats où les familles régnantes 
sont perçues comme les principaux entrepreneurs bénéficiant de l'importation de main-d'œuvre à bas coût, l'existence d'une large main-d'œuvre ségrégée dans le pays est plus problématique. C'est le cas du Bahreïn où la présence de migrants étrangers a exacerbé le ressentiment des nationaux à l'égard de l'affairisme de la famille royale (Louër 2008) ${ }^{12}$. C'est aussi le cas des EAU où la situation est moins tendue car les monarques y sont considérés par les citoyens comme les protecteurs de leurs privilèges face à leurs craintes de voir l'immense majorité d'étrangers prendre les rênes du pays (Herb 2009 : 392).

Dans ces derniers cas, la pérennité des politiques migratoires s'explique aussi par la logique purement économique qui, une fois la phase idéologique du nationalisme arabe passée, a présidé aux choix de recrutement de main-d'œuvre. Cette logique économique s'est retrouvée à l'unisson avec l'approche libérale qui s'est largement répandue après la guerre froide et qui fonde les parcours migratoires sur des bases aussi purement économiques.

La théorie des «systèmes migratoires ", qui prend en compte l'historique des relations entre pays pour expliquer la formation de réseaux migratoires, fournit ici des éléments importants pour expliquer le caractère décomplexé et purement économique avec lequel le CCG a pu appréhender les questions migratoires. Contrairement aux pays européens, qui doivent gérer leurs passés coloniaux, et aux États-Unis, qui doivent faire face aux conséquences de leur puissance hégémonique, la logique migratoire dans le Golfe est libérée de toute considération idéologique ou altruiste, même si elle ne l'est pas de considérations religieuses.

C'est la logique économique qui explique le fait, peu noté, qu'au fur et à mesure du développement des pays asiatiques le Golfe s'est tourné pour recruter ses migrants vers des origines toujours meilleur marché13. Cela vaut dans tous les domaines et a fortiori pour les emplois non qualifiés qui représentent la vaste majorité des emplois occupés par des étrangers. Le bas prix de la main-d'œuvre non qualifiée s'accompagne, selon les prémisses de l'économie libérale, d'un niveau très bas de compétences, y compris linguistiques. Ces carences sont structurellement compensées par l'effet de nombre notamment dans le domaine des emplois domestiques et les plus bas échelons des emplois de service (construction, restauration, etc.) ${ }^{14}$ - effet de nombre lui-même rendu possible par les prix bas du marché. Ce bas niveau de compétences, même compensé par l'effet de nombre, induit une faible productivité et concourt au renouvellement rapide de la main-d'œuvre, dans l'espoir d'obtenir de meilleures performances.

Dans ces conditions, la pérennité du modèle de migrations temporaires dans le Golfe doit être analysée non pas seulement en termes de restrictions de droits comme le fait une partie de la littérature existante, mais aussi, en termes de choix politiques, lesquels ne mettent pas en priorité le développement du capital humain.

41 En conclusion, la viabilité du modèle golfien d'import temporaire de main-d'œuvre étrangère est loin d'être remise en cause. Pour l'heure, la structure et la nature de l'emploi s'accommode, aux plus bas échelons, d'une main-d'œuvre sans cesse renouvelée et donc à faible rentabilité et, aux plus hauts échelons, s'inscrit dans les circuits de circulation des élites.

En replaçant les politiques golfiennes dans leur contexte historique, nous avons montré l'importance de la situation internationale comme facteur explicatif dans la région. Force est de constater que, depuis une vingtaine d'années, la libéralisation des 
mouvements et la mobilité accrue ont renforcé le système de rotation de la maind'œuvre dans le Golfe. Il a aussi été souligné le rôle joué par les nationaux - détenteurs du droit de la propriété immobilière et employeurs des migrants, et pas employés en compétition avec eux - dans la perpétuation du système.

L'adoption du modèle de rotation a permis d'éloigner les risques de déstabilisation des régimes liés aux migrants eux-mêmes et de mécontentement des nationaux en conservant un nombre élevé de main-d'œuvre bon marché et demandant peu d'investissement en capital humain. Du fait de la non-intégration des migrants, les nationaux ont tendance à les considérer en termes de biens autant que de personnes, et commencent à se demander à quelle partie du pacte social (famille royale, bourgeoisie marchande, classe moyenne) leur présence profite le plus, ce qui crée des tensions intérieures, cristallisées sur le fonctionnement du système rotationnel plus que sur son bien-fondé. Enfin, le faible investissement en capital humain qui caractérise le recrutement rotationnel golfien explique peut-être sa longévité qui risque le plus de la compromettre à terme.

\section{BIBLIOGRAPHIE}

ABELLA, M. I. 1995. « Asian migrant and contract workers in the Middle East », in R. COHEN (ed.), The Cambridge Survey of World Migration. Cambridge, Cambridge University Press.

ASMAR, M. R. [1990] 1995. The State and Politics of Migrant Labour in Kuwait. Leeds, University of Leeds.

BEAUGÉ, G. et A. ROUSSILLON. 1988. Le migrant et son double. Migrations et unité arabe. Paris, Publisud.

BIRKS, J. S., I. J. SECCOMBE et C. A. SINCLAIR. 1986. « Migrant workers in the Arab Gulf : The impact of declining oil revenues », International Migration Review, 20, 4 : 799-814.

BIRKS, J. S., I. J. SECCOMBE et C. A. SINCLAIR. 1988. « Labour migration in the Arab Gulf States : Patterns, trends and prospects ", International Migrations, 26, $3:$ 267-286.

BOGHARDT, L. P. 2006. Kuwait amid War, Peace and Revolution : 1979-1991 and New Challenges.

Basingstoke, Palgrave Macmillan.

CASTLES, S. 1986. « The guest-worker in Western Europe : An obituary », International Migration Review, 20,4:761-778.

CASTLES, S. 2006. « Guestworkers in Europe ; A resurrection? ? International Migration Review, 40, 4 : 741-766.

DADUSH, U. et L. FALCAO. 2009. « Regional arrangement in the arabian Gulf », International Economic Bulletin, juin : http://www.carnegieendowment.org/files/gcc1.pdf

FARGUES, P. 2000. Générations arabes. L'alchimie du nombre. Paris, Fayard.

FEILER, G. 1991. « Migration and recession : Arab labor mobility in the Middle East, 1982-1989 », Population and Development Review, 17, 1 (mars) : 134-155. 
FOX, J. W., N. MOURTADA-SABBAH et M. AL-MUTAWA (eds.). 2006. Globalization and the Gulf. Londres et New York, Routledge.

HERB, M. 2009. «A nation of bureaucrats : Political participation and economic diversification in Kuwait and the United Arab Emirates ", International Journal of Middle East Studies, 41, 3 (août) : 375-395.

KAPISZEWSKI, A. 2006. Arab versus Asian Migrant Workers in the GCC Countries. Beirut, Population Division, Department of Economic and Social Affairs, United Nations Secretariat.

LONGUENESSE, É., G. BEAUGÉ et M. NANCY. 1986. Communautés villageoises et migrations de main-d'œuvre au Moyen-Orient. Trois études d'anthropologie libanaise. Beyrouth, CERMOC.

LOÜ̈R, L. 2008. « The political impact of labor migration in Bahrain. », City \& Society, 20, $1: 32-53$.

MARCHAL, R. 2001. Doubaï : cité globale. Paris, Cnrs-Éditions.

ROUSSILloN, A. 1985. « Migrations de main-d'œuvre et unité arabe : les enjeux unitaires du modèle irakien », Tiers-Monde, 26, 103 : 637-664.

RUHS, M. et P. MARTIN. 2008. " Numbers vs. rights : Trade-Offs and guest worker programs », International Migration Review, 42, 1 (printemps) : 249-265.

RUSSELL, S. S. 1990. « Migration and political integration in the Arab World », in G. LUCIANI (ed.) , The Arab State, Londres, Routledge : 373-393.

SECCOMBE, I. J. 1983. «Labour migration to the Arabian Gulf : Evolution and characteristics 1920-1950 », Bulletin British Society for Middle Eastern Studies, 10 (1) : 3-20.

SECCOMBE, I. J. 1985. « International labor migration in the Middle East : A review of literature and research, 1974-84», International Migration Review, 19, 2 (été) : 335-352.

SECCOMBE, I. J. et R. I. Lawless. 1986. « Foreign worker dependence in the Gulf, and the International oil companies : 1910-50», International Migration Review, 20, 3 (automne) : 548-574

\section{NOTES}

1. Article de l'Arab Times, daté du 5 avril 2007 : «Immigration alien, present labour law shields expats; 'Changes to destabilize country », www.arabtimesonline.com.

2. Human Development Report 2009, A. Human movement: snapshots and trends, http:// hdrstats.undp.org/en/indicators/6.html.

3. Le système de sponsoring, ou kafala en arabe, bien que remis en cause récemment, perdure. Bahreïn a officiellement abandonné la kafala en 2009. Dans les faits, cependant, elle a seulement été réformée. Désormais, la délivrance des permis de travail est centralisée au sein d'un organisme administratif mais le lien exclusif entre employeur et migrant qui empêche ce dernier de changer d'emploi a été conservé. Le Koweït s'est récemment engagé dans la même voie avant de faire volte-face. « Kuwait sparks confusion with Labour U-turn », Financial Times, 16 octobre 2010.

4. En termes de droit du travail (représentation syndicale par exemple) et d'accès à la propriété.

5. Premier émirat indépendant en 1961, Koweït est aussi le mieux documenté, du fait de l'existence d'un parlement prévu par la Constitution de 1962, et celui dont la politique migratoire a été la plus influencée par l'idéologie intégratrice du nationalisme arabe - de sorte que son expérience pionnière a bénéficié aux autres États-cités du Golfe, qui s'en sont largement inspiré. 6. Détenteurs aux termes de la loi de $51 \%$ de l'entreprise implantée sur le sol koweïtien. 
7. Ceci vaut dans le cas de Koweït uniquement.

8. Les communautés iraniennes ou indiennes, dont l'implantation principalement dans les pays du sud du Golfe (EAU, Bahreïn, Oman) date de l'ère du pétrole, et qui n'ont jamais envisagé des mesures d'intégration ont, quant à elles, toujours conservé la sécurité de ces liens transnationaux.

9. Dans un article du quotidien koweïtien Awan, daté du 26 décembre 2009, les dirigeants de la banque islamique Kuwait Finance House se targuaient de ce que leurs emplois de direction étaient pourvus à $95 \%$ par des nationaux - fait partiellement remarquable seulement puisque les postes honorifiques de directeur, vice-directeur et membres du board des banques et entreprises du CCG sont toujours réservés aux nationaux.

10. Cette logique est validée par les résultats des études quantitatives de Ruhs et Martin (2008: 256) qui établissent une corrélation entre le nombre de travailleurs immigrés peu qualifiés accueillis dans un pays et les droits qui leur sont octroyés - même si les auteurs n'en tirent pas de conclusions prescriptives.

11. www.state.gov/g/tip.

12. La politique de taxation de la main-d'œuvre étrangère dans le secteur privé, dans l'espoir de réduire l'écart de productivité avec les nationaux, n'a fait que renforcer ce ressentiment.

13. Sans compter le possible recrutement que l'on ne peut qu'inférer des accords économiques signés en Afrique par le Premier ministre koweïtien Sheikh Nasser lors de son voyage sur le continent, en juillet 2009.

14. Voir par exemple les remarques faites par Dadush et Falcao (2009:5) « This gap in services productivity results in part from an overreliance on a relatively cheap and abundant stock of unskilled expatriate labor, on which the Gulf countries depend much more than other rich countries, due to their large resource endowment relative to their supply of native labor. Adopting a more efficient ratio of unskilled labor to other inputs in the service sectors that currently employ a high proportion of unskilled workers - for example, construction - would decrease reliance on imported unskilled labor. "

\section{RÉSUMÉS}

Si la majeure partie des études migratoires sont concentrées sur les migrations dites " NordSud», celles-ci ne constituent pourtant, d'après Peter Sutherland, représentant spécial des Nations Unies pour les migrations, qu'environ la moitié des flux migratoires mondiaux. Malgré les recherches poursuivies par les IFRE, qui notent que "certains pays d'émigration se sont transformés en terre d'immigration», ces migrations "Sud-Sud» restent moins documentées. Un «Sud» est assez particulier, à savoir les pays du Conseil de coopération du Golfe (CCG), qui, s'ils ne sont ni industrialisés ni ne se qualifient comme appartenant à une région avancée ou développée, comptent parmi les pays les plus riches du monde en termes de PIB per capita. Ce dernier indicateur met au jour deux éléments socio-économiques fondamentaux de la région : d'abord la grande richesse tirée des ressources en hydrocarbures, mais surtout, la population nationale de ces pays, peu nombreuse quand ils ont commencé leur envol économique, est restée proportionnellement faible, malgré les forts besoins en main d'œuvre engendrés par leur développement vertigineux et de leur forte natalité. Aujourd'hui, il ne fait mystère pour personne que dans certains pays du ccG qui, depuis leur création, sont tous des importateurs nets 
de main d'œuvre, la présence étrangère l'emporte en nombre sur la population nationale. Cette situation est le résultat de politiques délibérées visant à la non-intégration des populations immigrées. Cette logique de non-intégration défie beaucoup de propositions quasi-axiomatiques des études migratoires, dont le fer de lance a été la sociologie américaine préoccupée par les notions d'assimilation, de diversité ou multiculturalisme découlant d'une immigration considérée comme durable. Même lorsque le centre d'intérêt des études migratoires a été déplacé de l'analyse des conséquences de l'immigration vers celle de ses causes, ces postulats n'ont pas fondamentalement été remis en question comme en témoigne la théorie des systèmes migratoires et plus tard la littérature sur le transnationalisme qui présupposent la possibilité d'intégration dans le pays d'accueil pour pouvoir tisser des réseaux sociaux transfrontaliers. Même au regard du cas particulier de l'immigration turque en Allemagne, les politiques des pays du Golfe de refus d'implantation des étrangers alors même que leur présence est indispensable à l'économie du pays apparaissent assez singulières ; pourtant, malgré leur singularité au sein de la communauté internationale, elles ne semblent pas devoir changer pour des raisons structurelles comme l'a montré l'échec des politiques de nationalisation de la main d'œuvre dans leur objectif de réduire le déséquilibre entre nationaux et étrangers, et aussi du fait de la célébration assez générale de la mobilité des personnes et de la main d'œuvre dans le contexte de mondialisation. De fait, la montée en puissance du discours sur la mondialisation semble avoir opportunément fourni des justifications, dans deux domaines au moins, à des pratiques de ségrégation qui commençaient à être mise à l'index. Tout d'abord, les pays du CCG, l'émirat de Dubaï en tête, se sont présentés comme les champions de la mondialisation, avec leurs populations mêlées et leur position de carrefour : partisans du vieil adage libéral du "laissez-faire, laissez-passer », ils ont tâchés - pour les plus libéraux d'entre eux - de s'inscrire comme étape obligée dans les mouvements de circulation transcontinentale des élites, particulièrement occidentales (envisagées ici sous l'angle de migrations de travail seulement). En effet, la mobilité croissante de la main d'œuvre qualifiée et l'internationalisation des carrières semblent avoir fait passer au second plan la question de la non-intégration. Deuxièmement, le pilier de la politique d'immigration des pays du C CG depuis les années 1980 qui consiste à accueillir la main d'œuvre non qualifiée sur la base de contrats à durée limitée, semble avoir également gagné en acceptabilité avec la mise en place, pour adoucir les effets de la crise mondiale, par certains pays européens et le Japon de 'programmes de retour volontaire' des immigrants légaux, programmes qui attirent l'attention croissante des chercheurs. Dans le contexte de mobilité accrue, la tendance est-elle désormais à des migrations plus courtes des moins qualifiés, une rotation des élites, et une intégration sélective de certains immigrés triés sur le volet ? Assiste-ton à une relative convergence qui minimiserait la singularité du modèle du Golfe de nonintégration et le rendrait pionnier dans sa logique de déterritorialisation? L'analyse de la perception des phénomènes de migration et de mondialisation dans les discours et politiques des responsables du CcG, devrait nous permettre de répondre par la négative. Des mécanismes ont été mis en place au fil des années 1970 et 1980 pour assurer la non-intégration des étrangers dans les pays du CCG, depuis l'absence de naturalisation en dépit des lois, les politiques de régulation rigide des mouvements d'entrée, jusqu'au choix d'origine des travailleurs étrangers; ce principe de non-intégration est à la base du déni officiel du phénomène d'immigration par les autorités, mais aussi de la structure compartimentée et hiérarchisée des sociétés de la région. Le discours hégémonique de la mondialisation a eu un effet de trompe l'œil sur la justification et la rationalisation d'un modèle résultant de politiques le plus souvent à court terme. Sa viabilité à long terme et les conditions de son évolution sont étudiées à travers le cas de Bahreïn qui, parent pauvre du CCG, a commencé timidement à revenir sur certaines rigidités du système en termes de nationalité et de sponsorship. 
Most migratory studies focus on "North-South" migration. However, according to Peter Sutherland, the United Nations' Special Representative on migration, these migrants only account for around half of global flows. Despite research carried out by the French Research Institutes Abroad (Instituts français de recherche à l'étranger or IFRE) indicating that "some emigrant countries have now become host countries," there is much less information on "SouthSouth" migration. One "Southern" country stands out: the Cooperation Council for the Arab States of the Gulf (GCC). Even though they are industrialised and do not belong to advanced or developed regions, they are some of the richest countries in the world in terms of GDP per capita. This highlights two socio-economic factors unique to the region: firstly, the wealth generated by oil resources and, secondly, the fact that these countries, which had small populations when their economies began taking off, have remained relatively unpopulated despite their booming economies, strong demand for labour and high birth rates. It is no surprise that in some GCC countries, which have been net importers of labour since the GCC's creation, there are more foreigners than citizens. This is because of policies deliberately aiming at the non-integration of immigrant populations. This non-integration goes against many near axiomatic conclusions from migratory studies, in particular American sociology, which has focused on the concepts of assimilation, diversity, and multiculturalism as a result of enduring immigration. Even when migratory studies began emphasizing the causes of immigration rather than its consequences, these fundamental ideas were never seriously called into question. This can be seen in the migrations system theory and work on transnationalism, which assumed that immigrants could integrate host countries and build cross-border social networks. Even compared to the unusual situation of Turkish immigrants in Germany, the fact that Gulf countries have adopted policies preventing foreigners from settling in the region - despite they fact are essential to the economy - can seem strange. However, even though these policies are unique in the international community, they do not seem to require modification for structural reasons including the failure of policies nationalising labour to reduce the imbalance between citizens and foreigners, and the generalised position encouraging personal and labour mobility as a result of globalisation. In fact, the increasing importance of arguments in favour of globalisation seems to have justified segregationist practices in at least two fields that were beginning to be frowned upon. Firstly, the GCC and Dubai in particular set themselves up as the champions of globalisation, with their mixed populations and strategic geographic locations. As supporters of the old "laissez-faire, laissez-passer" saying, the most liberal countries attempted to become key ports of call in the movement of a transnational - especially Western - elite (considered solely in the context of labour migration). Indeed, the increasing mobility of qualified labour and the internationalisation of careers seem to have pushed the issue of non-integration into the background. Secondly, the foundation of the GCC's immigration policy since the 1980s - fixed term contracts - seems to have become more acceptable, with the implementation of "voluntary return programmes" for legal immigrants in Japan and some European countries. Introduced to mitigate the effects of the economic crisis, these programmes are attracting the attention of researchers. Given this increased mobility, what are the current migratory trends: shorter migration periods for less qualified workers, a rotating elite and the selective integration of a few handpicked immigrants? Are we currently witnessing a situation where different situations converge to minimise the peculiarity of Gulf countries' non-integration models and make them pioneers in the de-territorialisation field? An analysis of the description of migration and globalisation in GCC speeches and policies indicates this is not the case. In the 1970s and 1980s, the GCC put in place several measures preventing the integration of foreigners. This included the non-naturalisation of foreigners despite laws to the contrary, strict policies controlling foreign admission to the country, and accepting foreign workers from selected countries only. The nonintegration principle underlies the authorities' official position denying immigration, but also the compartmentalised and hierarchical structure of societies in the region. The hegemonic 
discourse on globalisation has had an illusory effect on the justifications and rationalisations for a model resulting from what are mostly short-term policies. Its long term viability and possible evolutions are examined by taking the example of Bahrain, one of the GCC's poor relations, which has begun to timidly revise some of its strict provisions on nationality and sponsorship.

INDEX

Thèmes : mobilité, migration

Keywords : system of non-integration, immigrant labor, temporary migration, Saudi Arabia, Bahrain, UAE, Kuwait, Qatar, Oman

Index géographique : Arabie Saoudite, Bahreïn, Émirats arabes unis, Koweït, Qatar, Oman

Mots-clés : système de non-intégration, travail immigré, migration temporaire

\section{AUTEUR}

\section{CLAIRE BEAUGRAND}

Doctorante en Relations internationales, London School of Economics and Political

Science, elle a été allocataire au Centre français d'archéologie et de sciences sociales de Sana'a (CEFAS-UMIFRE 5), http://www.cefas.com.ye/, en 2005 et 2006

c.b.beaugrand@lse.ac.uk 